Ilie Mihaela, Nedelcu Cristina, Ulmeanu Victoria, Tuta Liliana, Scrinic Olesea, Muflic L., Oanta M.,

\begin{abstract}
Acute pancreatitis represents the acute inflammatory process of the pancreas which can remain localized in the gland or could extend in the tissues around the pancreas or retroperitoneal tissues and also at the organs from the pancreatic lodge.

The diagnosis is usually based on the increase in pancreas volume and on the changes in structure objected on the ultrasound examination. It is characterised by a "release" of enzymes out of the pancreatic ducts associated with oedema and necrosis in the parenchyma and in the retroperitoneal area.
\end{abstract}

Keywords: acute pancreatitis, ultrasonography, peripancreatic fat necrosis

\section{Liliana Tuta}

Faculty of Medicine, "Ovidius" University of Constanta,

Nephrology Clinical Service, Saint Apostle Andrew Emergency County Hospital, 145 Tomis Avenue, 900591

phone: +40722300505

email: tutaliliana@yahoo.com

\section{Introduction}

Acute pancreatitis represents the acute inflammatory process of the pancreas which can remain localized in the gland or could extend variably in the tissues around the pancreas or retroperitoneal tissues and also at the organs far from the pancreatic lodge[1].

Acute pancreatitis is a disease in which ultrasonography is absolutely useful for diagnosis establishment and therapy guidance[2].

\section{Objectives}

The characterization from a clinical and echographic point of view, of the patients which have 
acute pancreatitis

Survey of the echographic sensibility in establishing a positive diagnosis of acute pancreatitis

\section{Materials and Method}

Retrospective survey

Period of the survey: January 2013 - June 2014

Group of 60 hospitalized patients in Medical Clinic II with acute pancreatitis

There were extracted the clinical and biological data, and the results of the ecographic exploration.

There was calculated the sensibility of the diagnosis method

\section{Results}

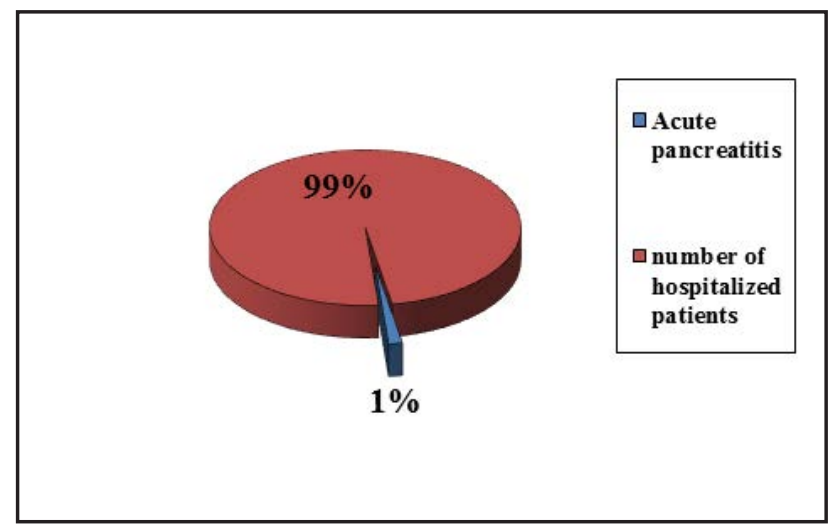

Frequency of pancreatitis in comparison with the total number of hospitalized patients

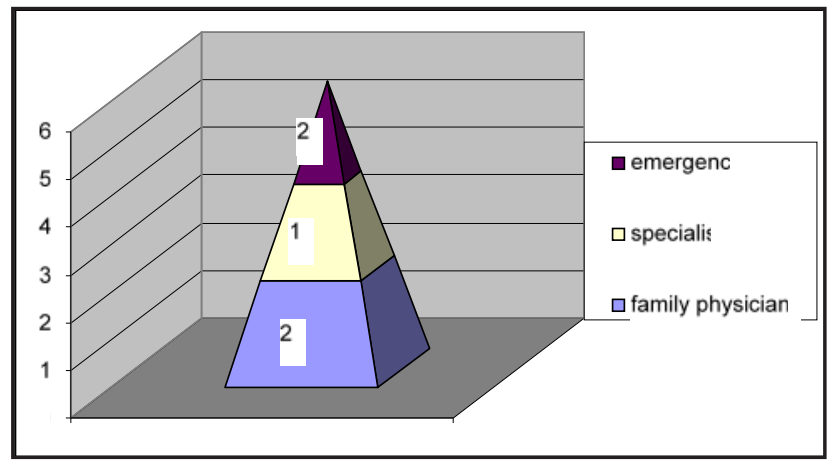

The modality in which the patients were hospitalized

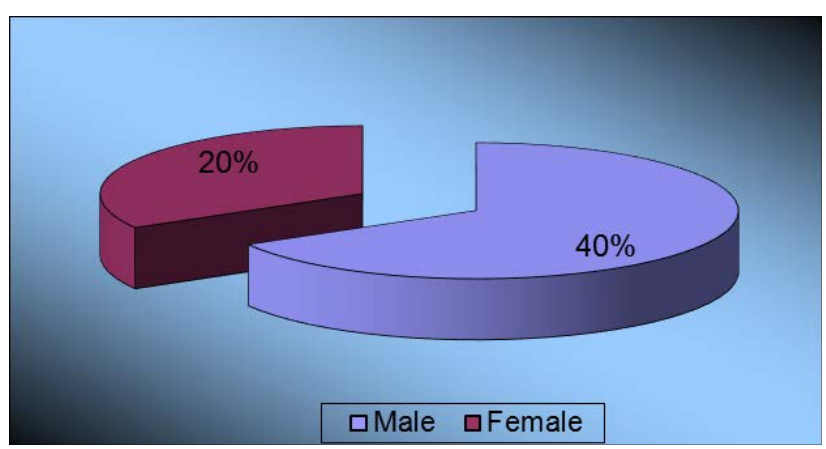

Distribution of cases regarding the patient's sex

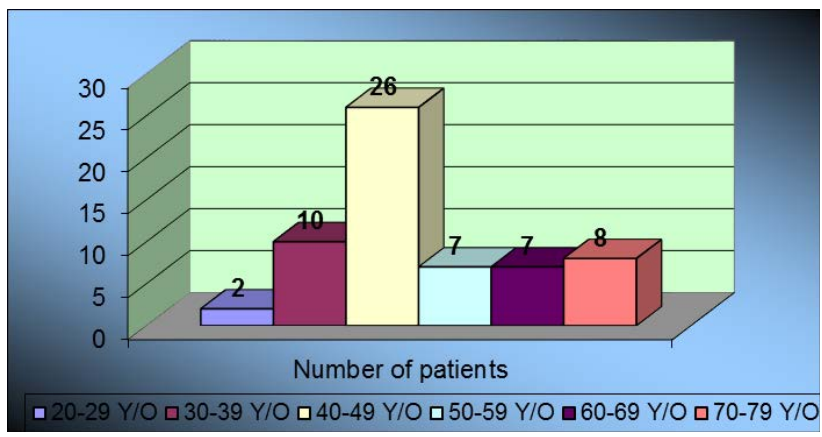

Distribution of cases in respect of the age group

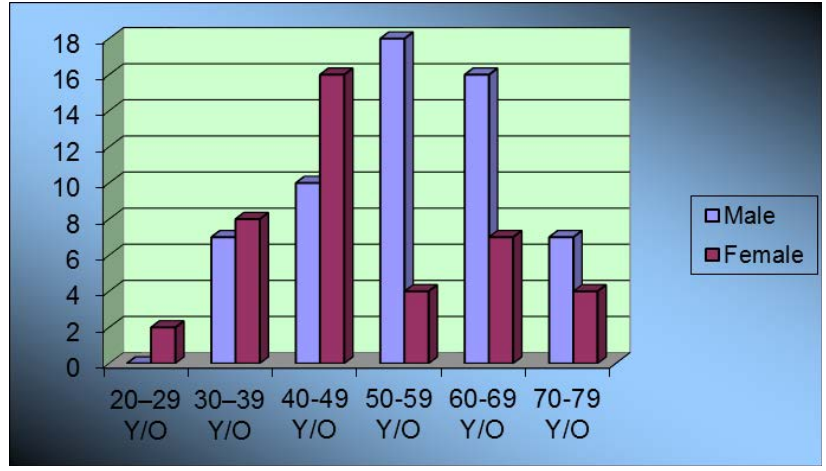

Distribution of cases in respect of age group and gender 


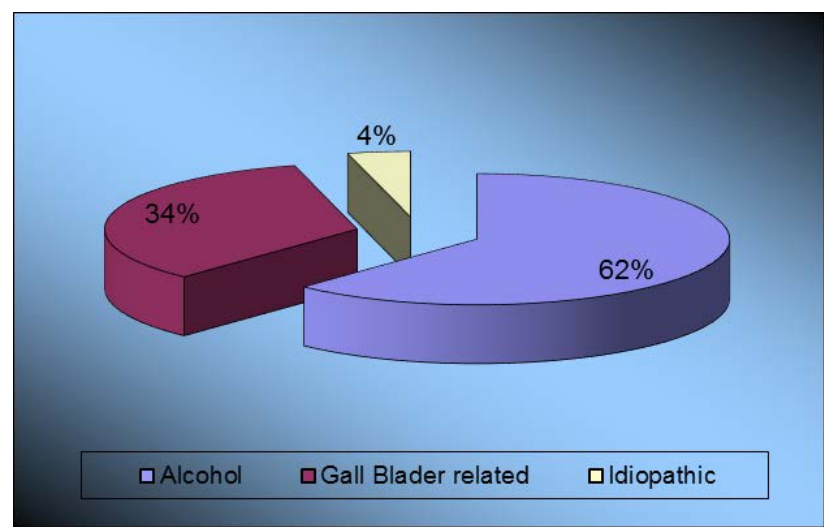

Distribution of cases in respect of etiology

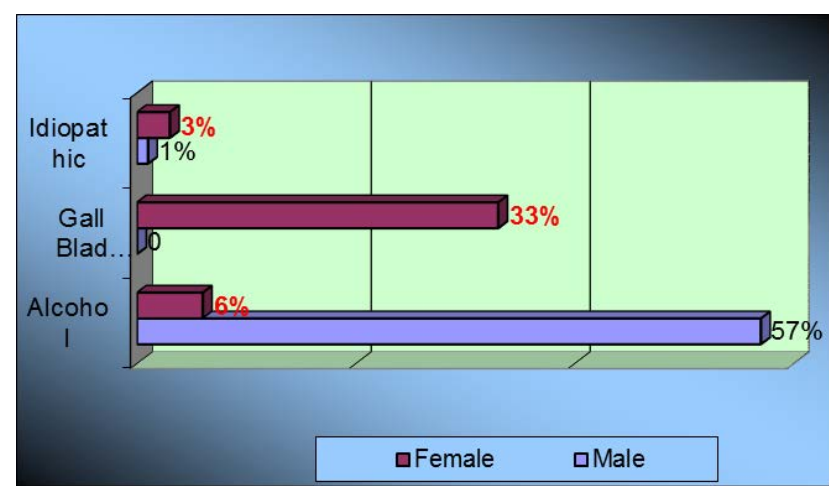

Distribution in respect of etiology and gender

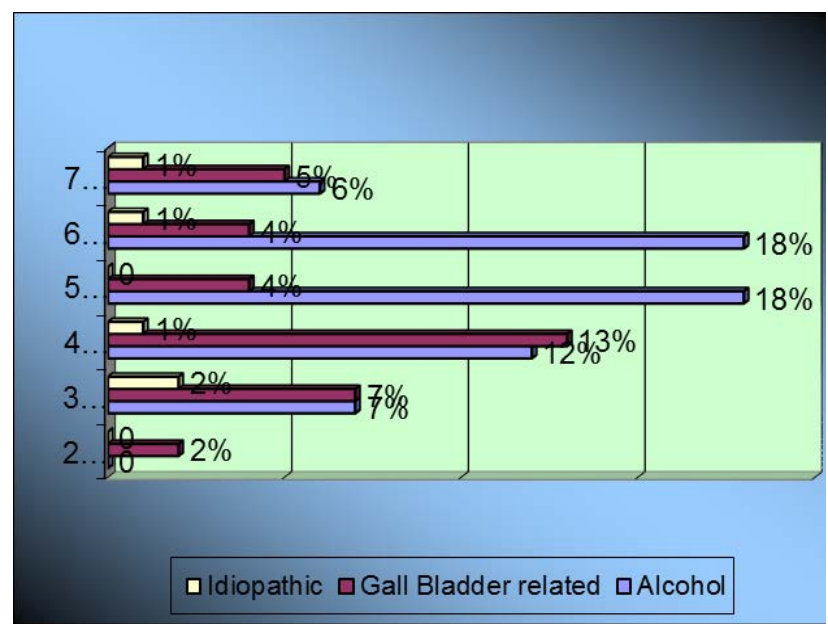

Distribution of cases in respect of etiology and age group

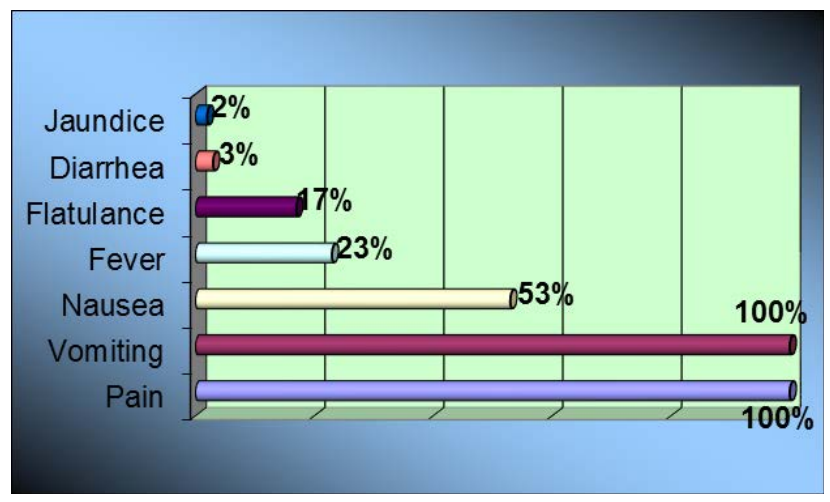

Distribution of cases in respect of the internment symptomatology

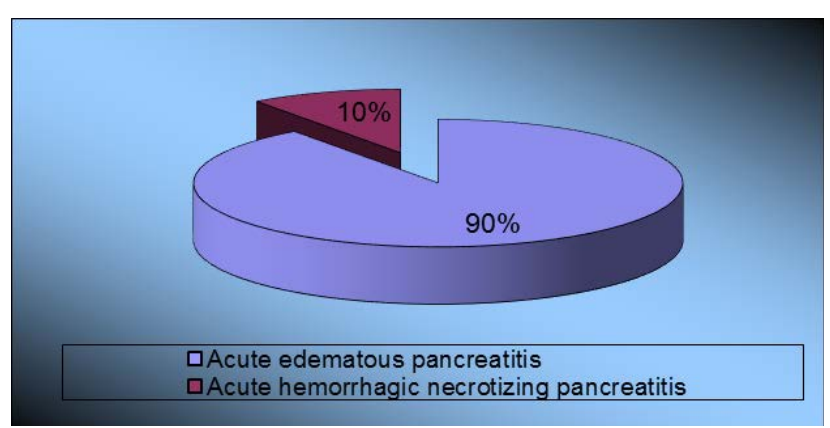

Distribution of cases in respect of the clinical form

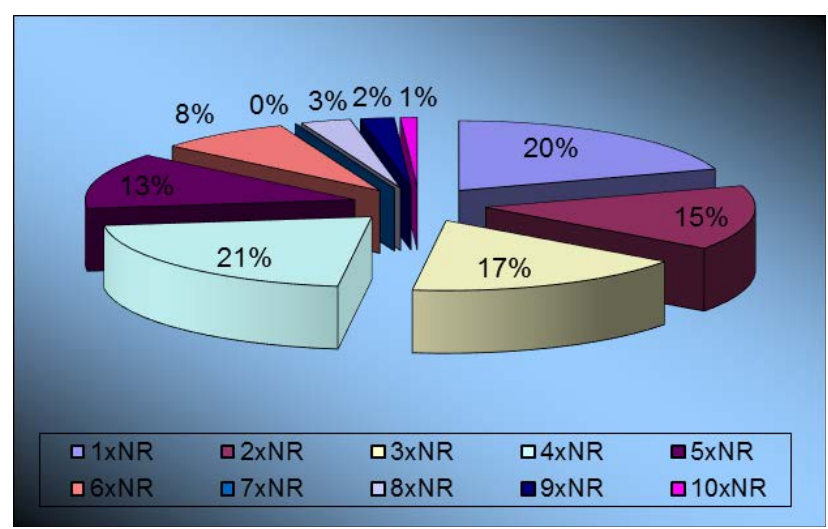

Distribution of cases in respect of the analysis of serum pancreatic amylase at internment 


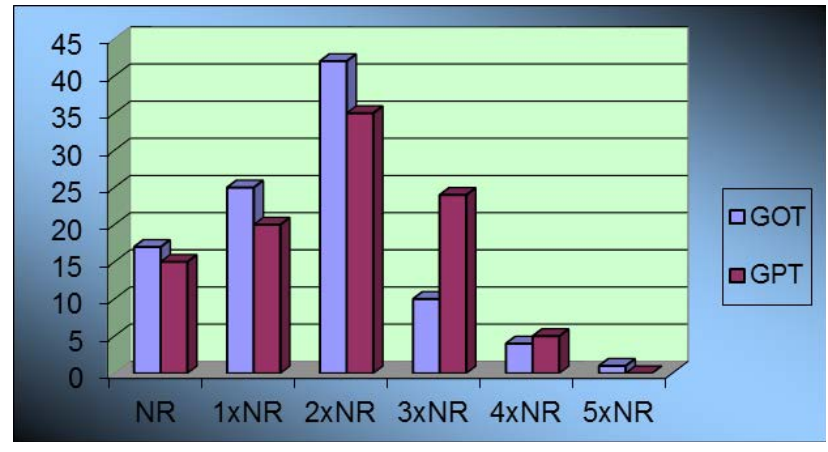

Distribution of cases in respect of GOT/GPT values at internment

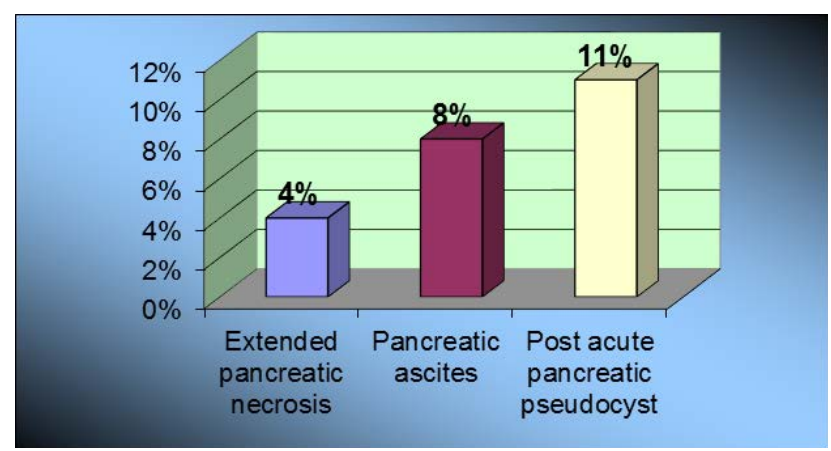

Distribution of cases in respect of complications

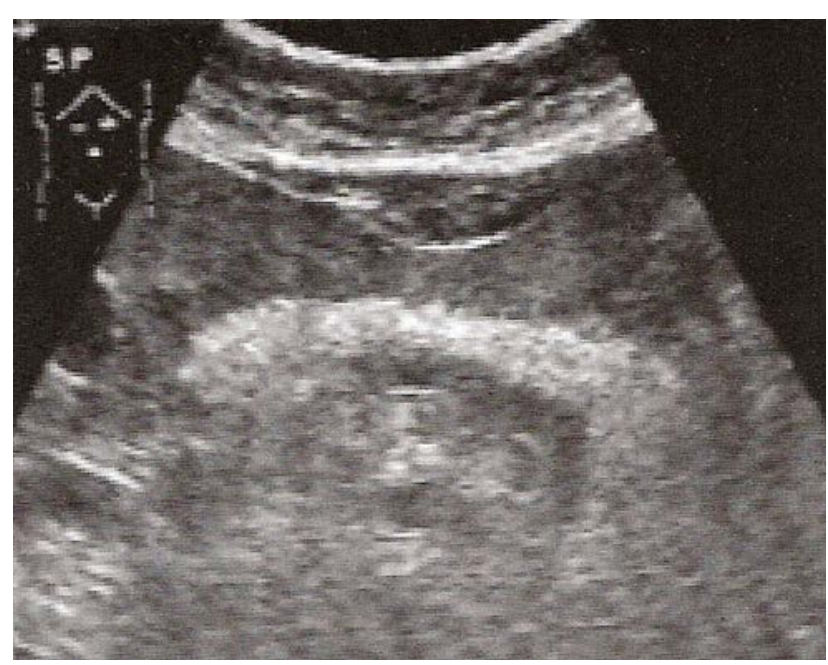

Normal ecographic aspect of the pancreas, Overweight patient

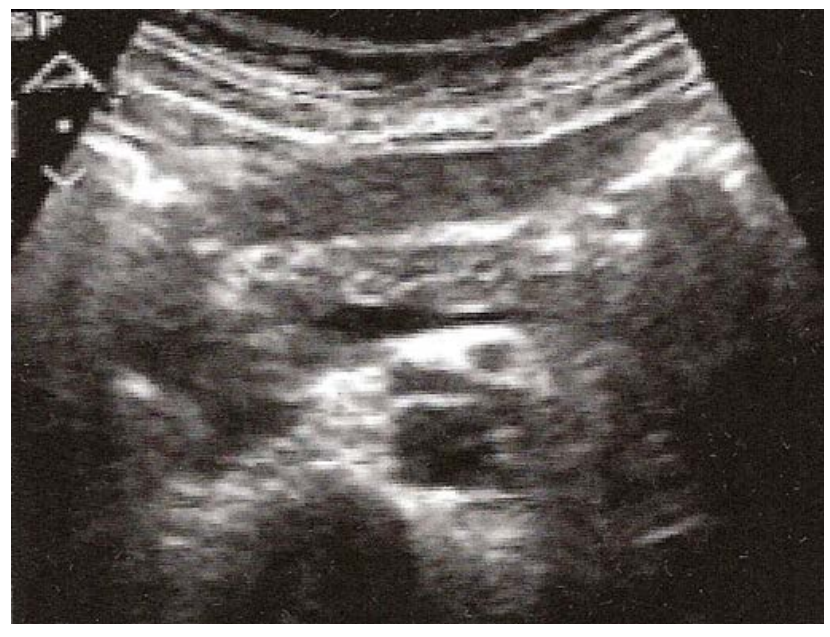

Normal ecographic aspect of the pancreas, Normal weight patient

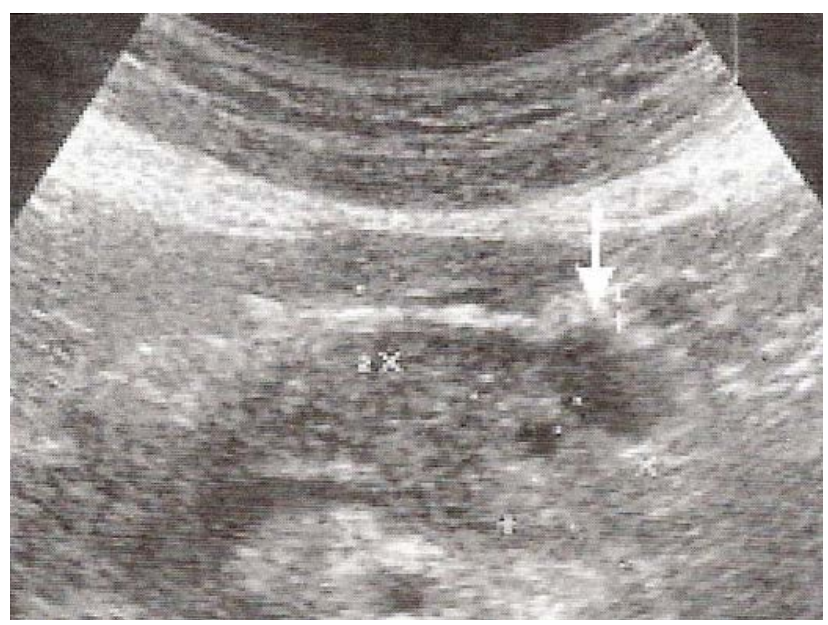

Acute pancreatitis: peripancreatic fat necrosis

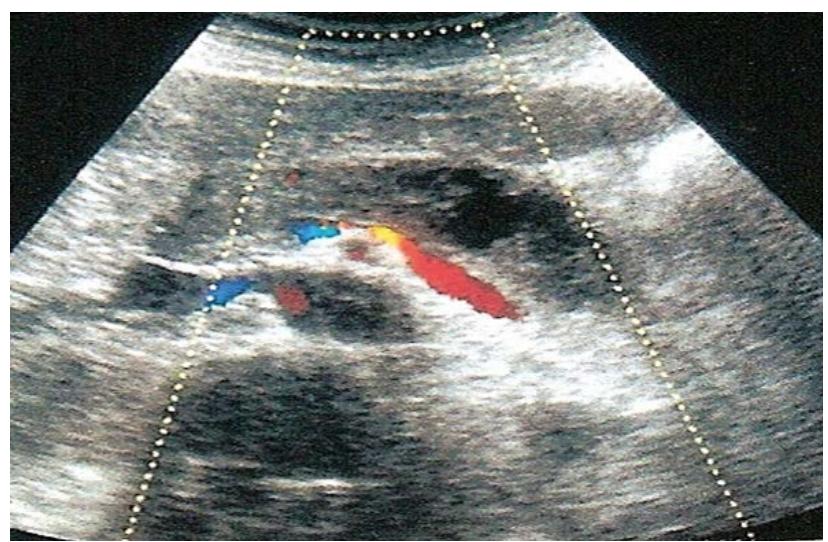

Acute necrotizing pancreatitis (body-tail zone) 


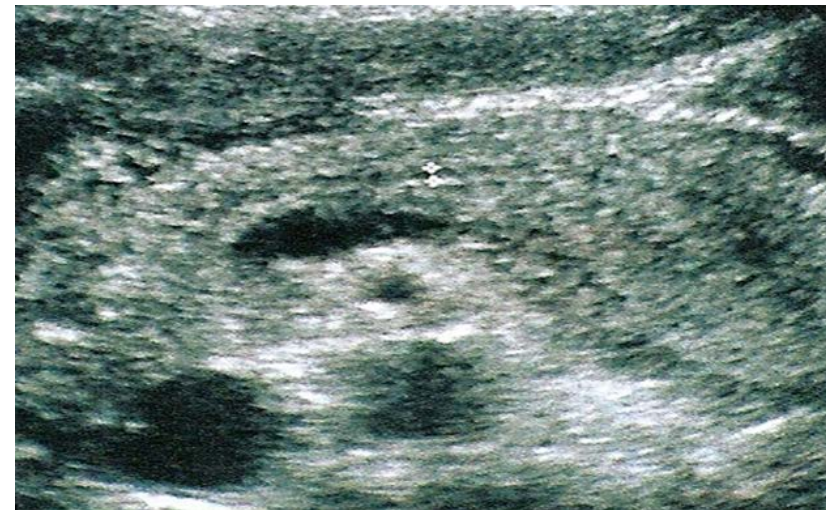

Acute pancreatitis (peripancreatic ventral liquid accumulation; tail with increased volume)

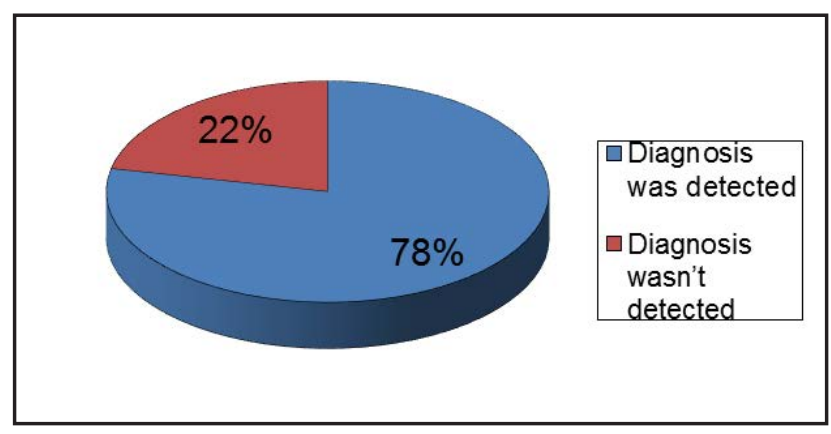

Contribution of ecography in acute pancreatitis diagnosis

\section{Conclusions}

Acute pancreatitis represents $1 \%$ from the total hospitalizations in the Medical Clinic II of the Clinical County Hospital Constanta, recording 60 cases in the 2013-2014 periods, with an increased incidence in males.

Males are more affected in all age groups, acute pancreatitis being more frequent at women in the age group of 40-49 years old, and the incidence is relatively equal at both sexes in the ages comprised between 30-39 years old.

The most frequent etiology of acute pancreatitis is alcohol $-62 \%$ of cases and it prevails in men (commentary: acute gallbladder-pancreatitis cases diagnosed in the emergency service are hospitalized in the Surgery Clinic). Regarding the women the most frequent etiology is gallstones.

As an etiological factor, alcohol prevails at the ages between 50 and 69 years old, and gallstones at young ages (20-29 years old and 40-49 years old).

The main symptoms met were abdominal pain and vomiting (100\% of cases); diarrhea and jaundice were associated with severs form of the disease.

$90 \%$ of the studied patients were diagnosed with acute edematous pancreatitis, and $10 \%$ with acute hemorrhagic necrotizing pancreatitis.

Amylasemia has lack of specificity and reduced sensibility ( $35 \%$ of the patients have $<3 \times$ NR insignificant for the diagnosis) approximately $60 \%$ of the subjects were associated with hepatocyte lysis syndrome.

Acute pancreatitis complications of the patients under investigation were rare, extended pancreatic necrosis- 4 cases, pancreatic ascites -8 cases, pancreatic pseudocyst -11 cases.

Abdominal ecography was conducted at all patients and was the investigation that oriented the diagnosis, having $80 \%$ accuracy for the positive diagnosis of acute pancreatitis.

The advantages of ecography are: it is a noninvasive exploratory method, cheap, nonirradiated, and reproducible and allowed the tracking of the patients with severe forms and complications that in the end were guided to the surgery clinic.

Abdominal ecography is indicated for the diagnosis of all forms of acute pancreatitis.

\section{Acknowledgement}

This work received financial support through the project entitled "CERO - Career profile: Romanian Researcher", grant number POSDRU/159/1.5/S/135760, cofinanced by the European Social Fund for Sectoral Operational Programme Human Resources Development 20072013. 
1. Badea, R. (1995). Ultrasonographic changes in acute pancreatitis. Romanian Journal of Gastroenterology. 14(1), 83-89

2. Jeffrey, R.B. \& Ralls, P.W. (1995). The liver in sonography of the abdomen. New York: Raven Press 